CORRECTION

https://doi.org/10.1038/s41586-018-0285-x

\title{
Author Correction: A massive core for a cluster of galaxies at a redshift of 4.3
}

T. B. Miller, S. C. Chapman, M. Aravena, M. L. N. Ashby, C. C. Hayward, J. D. Vieira, A. Weiß, A. Babul, M. Béthermin, C. M. Bradford, M. Brodwin, J. E. Carlstrom, Chian-Chou Chen, D. J. M. Cunningham, C. De Breuck, A. H. Gonzalez, T. R. Greve, J. Harnett, Y. Hezaveh, K. Lacaille, K. C. Litke, J. Ma, M. Malkan, D. P. Marrone, W. Morningstar, E. J. Murphy, D. Narayanan, E. Pass, R. Perry, K. A. Phadke, D. Rennehan, K. M. Rotermund, J. Simpson, J. S. Spilker, J. Sreevani,

A. A. Stark, M. L. Strandet \& A. L. Strom

Correction to: Nature https://doi.org/10.1038/s41586-018-0025-2, published online 25 April 2018.

In this Letter, the Acknowledgements section should have included the following sentence: "The National Radio Astronomy Observatory is a facility of the National Science Foundation operated under cooperative agreement by Associated Universities, Inc.". This omission has been corrected online. 